\title{
EVALUACIÓN, ACREDITACIÓN, CALIDAD EDUCATIVA E IPEBA: Cuidado con las utopías, la matriz y la imposición ${ }^{1}$
}

\author{
Dr.Eduardo PalominoThompson
}

\section{RESUMEN}

Ante la gran expectación y esperanza suscitada en el Perú por el inicio del Sistema Nacional de Evaluación, Acreditación y Certificación de la Calidad Educativa (SINEACE), el autor revisa los lineamientos legales y normativos referidos al SINEACE y al IPEBA y su matriz de gestión (2011). Estando de acuerdo con lo positivo del sistema de Evaluación y Acreditación, procura poner en guardia ante un al parecer excesivo optimismo que estaría llevando a una situación utópica, lo cual podría luego transformarse en una frustración educativa nacional.

Esta preocupación aumenta al comprobarse, ante el análisis legal y normativo que se hace, que el esquema evaluativo propuesto por el IPEBA no aparece como integral, ya que se concentra, sobre todo, en la evaluación de la gestión, dejando de lado aspectos de los aprendizajes relacionados con lo cognitivo y no cognitivo.

Por otra parte, el autor insiste en que el SINEACE y sus organismos no pueden garantizar la calidad educativa, como se deduce del marco legal y normativo, sino colaborar a promoverla.

Asimismo, recomienda respetar la libertad de enseñanza $y$, en esta línea, permitir que las instituciones educativas, de acuerdo a su propia cosmovisión, puedan establecer para la Evaluación y Acreditación, algunos estándares, factores o criterios e indicadores propios, y no estar así sometidos únicamente a lo señalado al respecto por el Estado mediante el IPEBA, organismo para la Educación Básica, nivel al que se refiere este trabajo.

Nada de esto quita el muybuen aporte que significa el trabajo del IPEBA, el cual ha producido documentos orientadores significativos y que suponen un gran esfuerzo ydedicación.

\section{ABSTRACT}

In view of the great expectation and hope in Peru caused by the launching of the National System of Evaluation, Accreditation and Certification of the Ouality in Education (SINEACE), the author reviews the legal dispositions and norms referring to SINEACEANDIPEBAand itsmanagement guidelines (2011). Agreeing with the success of the Evaluation and Accreditation system, he calls the attention to an apparent and excessive optimism which could be leading to a utopian situation transforming then to a national educational frustration.

The concern increases when faced with a legal and standardized analysis, the evaluative scheme proposed by IPEBA is not shown as a whole evaluation because it is chiefly concentrated in management evaluation, leaving apart cognitive and non cognitive learning aspect s.

In the other hand, the author insists in that SINEACE and its components do not warranty the quality in education, as it is deduced from the legal and regulatory framework, but helps to promete it.

Furthermore, he recommends the free teaching and in the same way, let educational institutions according to their own view, to fix sorne own standards, factors or criteria and indicators for Evaluation and Accreditation, and not being under what it is recommended by the State through IPEBA, organism for Basic Education.

Nevertheless, we can saythat IPEBA's work is very significant, and has produced guiding documents as a sign of effort and dedication

\section{PALABRAS CLAVE}

Calidad integral, utopía, aprendizajes cognitivos, aprendizajes no cognitivos, gestión institucional.

\section{KEYWORDS}

Whole quality, utopía, cognitive learning, non-cognitive learning, institutional management. 


\section{l. INTRODUCCIÓN}

A nte todo, en este artículo no se cuestiona el valor de la evaluación, autoevaluación y acreditación para mejorar la calidad de la educación; tampoco el valioso y esforzado trabajo del IPEBA, organismo del SINEACE encargado de la Básica Regular, Lo que se desea es señalar con claridad aquello que se considera inadecuado, parece incompleto, u otros, a fin de que estos importantes procesos sean exitosos. Asimismo:

a) Que no se conviertan en una "utopía peligrosa" y en una de las tantas frustraciones educativas nacionales.

b) Que se tenga siempre un enfoque educativo integral, y no reductivo, que ayude a que los verificadores sean elementos realmente positivos e integrados, sin pensar que son los únicos o los principales, ya que hay otros aspectos educativos de muy difícil verificación.

c) Que la necesaria libertad de enseñanza y la autonomía de los centros no disminuya, o se viole por exigencias constrictivas debido a la aplicación de parámetros exagerados.

Es evidente que el mundo educativo, con los resultados exitosos o no exit osos $^{2}$ de los estudiantes, constituye una de las tramas más complejas del quehacer humano.

En lo que corresponde a la preocupación por la calidad educativa, el hombre y los Estados, en su pasión sistematizante y matematizante, más aún en esta época de estándares, variables, indicadores, y otros, pretenden con frecuencia, vano intento por cierto, hacer un camino casi perfecto ${ }^{3}$ hacia logros educativos de calidad, planteando que todos los estudiantes e instituciones pasen por una vía concreta preestablecida por ellos (estándares, factores, indicadores etc), bajo la razón de que, finalmente, solamente así se obtendrá un producto de "calidad garantizada" que comprenda por igual a todas las instituciones y estudiantes a un nivel de alguna manerasimilar,

En cierto sentido, esta inquietud y sueño es válido; es más, son los sueños los que han conducido a las grandes utopías y realizaciones. Las utopías, las sensatas, han hecho un gran bien a la humanidad y han permitido avances sustanciales.

Pero también es cierto que, las utopías exageradas, han producido males ampliamente certificados por la historia . En el caso de la educación en el Perú, esto podría acontecer en lo referente a la Evaluación, Acreditación, Calidad Educativa y los organismos del SINEACE si no se van analizando, y en casos rectificando, los pasos que se van dando, o si se exageran las expectativas.

Por otra parte, es posible que estemos también ante una "utopía educativa algo equivocada" desde otro punto de vista: el de un enfoque reductivo de la calidad, ya que la matriz de gestión del IPEBA presentada (2011), se concentra precisamente en la gestión y prescinde de otros aspectos sustantivos, hecho que podría determinar (si no estamos ya en esta situación) medidas y/u orientaciones que, lejos de ayudar, desvíen de logros educativos integrales deseados por todos. (Pudiera ocurrir también que más adelante salga otra documentación que mejore esta situación).

De otro lado, es notorio que en estos esquemas de evaluación modernos, se da una importancia casi sagrada a "verificadores" básicamente muy puntuales y cuantitativos. Pero, ¿de dónde parte esta visión de darles un valor, tal vez exagerado, a estos "verificadores" (evaluación, autoevaluación, acreditación)? Tal vez se podría hablar de al menos cuatro puntos de partida:

a) Como es frecuente, se inicia al parecer por la tendencia humana a buscar soluciones medio "mágicas" a problemas complejos. Lo hemos visto con frecuencia a través de nuestra historia educativa. En efecto, pensábamos que con algunas estimables novedades se arreglaba todo; por ejemplo, una nueva Ley de Educación, el Proyecto Educativo Nacional, cambios en el diseño curricular, variar el sistema de evaluación escolar, cambiar la teoría de aprendizaje del momento, idear un nuevo modelo de programación y otros muchos etcéteras. Todo ha ayudado, pero ninguno lo ha resuelto. Es que no existen soluciones únicas ni mágicas.

b) No cabe duda que esta visión optimista hacia algunos medios educativos como los mencionados, parte muchas veces de gobiernos y autoridades que, por réditos políticos, exageran el valor de los nuevos planteamientos que están liderando, tratando de hacer creer que en ellos está la tan soñada solución educativa.

c) Otro punto de partida del afán por las soluciones únicas y mágicas es ciertamente la documentación oficial (leyes, reglamentos o normativas) que establece la nueva orientación. 
Se trata en este caso de: aspectos correspondientes de la Ley General de Educación; ley y reglamento del SINEACE; guías y matrices concretas de estándares, criterios, indicadores, etc. Para cualquier caso, se hace necesario revisar sí dicha documentación, por lo que establece, no está impulsando a una "utopía exagerada" que ve, en la realización normada, la solución siempre soñada. Con frecuencia, se quiere garantizar la calidad educativa mediante la multiplicación dedocumentos einstrumentos, cosaquemásbiendificulta ellogrodeseado.

d) Por otra parte, dentro del ordenamiento menor, pero importante y clave, habrá que revisar sí es que existe allí un exceso en los detalles de las normas; sí ello mismo no lleva a una documentación muy constrictiva y vertical Caricaturizando: "aquí te doy los estándares, factores, indicadores, etc.; ellos son los que llevan a la calidad educativa que queremos; tienes que seguirlos, no hay otros". Obviamente, no se debe tratar de determinarlo todo desde la autoridad, no solamente porque ello implica quebrar la garantía constitucional de libertad de enseñanza, sino por lo ilógico y perjudicial de querer normar contextos y situaciones culturales tan diferentes y detalles que manejan mejor las instituciones.

\section{PRESUPUESTOS BÁSICOS DE ESTAS REFLEXIONES}

a) La amplitud del tema (teoría que lo sustenta y realizaciones prácticas del mismo) hace que se planteen solamente: A) algunas reflexiones y observaciones en torno a aspectos legales y normativos ( $\left.\mathrm{N}^{\circ} 4\right)$ que fundamentan el tema, los cuales son ciertamente el punto de partida con el que se están desarrollando actualmente nuestros procesos de Evaluación, Autoevaluacíón y Acreditación (a los que en diversos momentos denominamos verificadores); B) se plantean observaciones a aspectos teóricos y prácticos de la matriz de evaluación para la Acreditación presentado por el IPEBA en mayo de 2011 ( $\left.\mathrm{N}^{\circ} 5\right)$; C) se termina con unas Conclusiones Generales $\left(\mathrm{N}^{\mathrm{o}} 6\right)$, con Recomendaciones ( $\mathrm{N}^{\circ}$ 7) y Referencias ( $\left.\mathrm{N}^{\circ} 8\right)$.

b) Es necesario señalar desde el primer momento que, en los hechos, la educación peruana está tomando los términos evaluación, autoevaluacíón, acreditación y competencia prof esíonal en un sentido predominante de gestión, dejando muy marginadas las dimensiones espirituales en el amplío sentido de la palabra (persona, cultura, valores, ética, religión), así como la verificación de aspectos cogmnvos. La orientación puede ser muy peligrosa y traer serías consecuencias para la educación y el desarrollo integral de las personas y de la sociedad, ya que reduce sustantiva e indebidamente el campo a verificar y se aparta de una educación integral. La matriz presentada por el IPEBA pertenece, en opinión del autor, a esta orientación, aunque hay que tener presente que, cuando aparezcan estándares más concretos, esto podría mejorar.

\section{REFLEXIONES PREVIAS}

a) Lo primero que exige la calidad es que la base teórica del Proyecto Educativo de las instituciones sea sólida, integral, con un marco educativo intelectual que responda a un concepto de educación acorde con los mejores planteamient os. Asimismo, que vaya a la totalidad delos estudiantes.

Sí la base teórica es deficiente, por más que los verificadores (evaluación, autoevaluacíón y acreditación) sean técnicamente impecables, los resultados educativos no podrán ser óptimos, aunque aparentemente lo sean por responder a los fines, objetivos y metas propuestas por la institución educativa. Es decir, no basta que se haya obtenido un buen resultado en lo proyectado sino, ante todo, que lo proyectado sea bueno: hay que ver "cuán bueno es el proyecto". La evaluación y acreditación dan resultados y certifican, pero no garantizan la calidad del proyecto.

b) Por otra parte, estambién necesario queelmodelo teórico que emplea el IPEBA en su esquema de evaluación y acreditación sea el adecuado y, entre otros, no tenga vacíos importantes que puedan hacer que la educación sea orientada por un camino ind ebido. Sí por ejemplo, en la concreción de lo escogido como factores, estándares e indicadores, no están los mejores, o falta alguno importante como por ejemplo lo ético o los valores, el esquema evaluador y acredítador será deficiente y hará que las instituciones educativas es col a res sean impulsadas a seguir un camino equivocado; un camino que no es el de la calidad integral ya que conduce parcialmente a ella.

Tampoco podrían admitirse fallas en la 
jerarquización, otorgando mayor importancia a aspectos que el consenso considera no prioritarios.

En consecuencia, y sea esta una importante observación, la calidad no queda asegurada por una buena evaluación, autoevaluación y acreditación; el proyecto debe ser realmente de calidad teórica, tanto en la institución educativa como en los planteamientos matrices del IPEBA.

Esto quiere decir que el esquema teórico debe estar asentado en la Constitución del Perú que manifiesta un sentido integral. Así, justamente el artículo 13 que inicia la parte educativa dice: "La educación tiene como finalidad el desarrollo integral de la persona humana". Es decir, si existen aspectos importantes ausentes, el cantina no es el adecuado.

c) Lo tercero que parece sustantivo es: cuán técnicamente elaborados están el esquema y los instrumentos de evaluación aplicados, así como los indicadores utilizados, sea por disposición del IPEBA, por la institución, o por la agencia acreditadora. En efecto, si los indicadores no verifican lo más importante porque están deficientemente elaborados (lo técnicamente sólido), no se puede asegurar si en la institución existe calidad educativa.

d) Por otra parte, y como último aspecto previo, hay que afirmar que un conjunto de distintos indicadores, diversos factores o ejes, y estándares, no garantizan la calidad educativa, la cual es algo bastante más amplio. Ellos no indican, ni de lejos, la riqueza de una institución o los valores de una personalidad en el caso de los estudiantes, más aún cuando los indicadores, criterios, estándares, y otros, se suelen concentrar en aspectos numéricos, administrativos, la gestión, la profusión de documentos, infraestructura, esquemas organizativos,etc.

La verdadera calidad va mucho más allá. Sin negar el aporte de todo lo anterior, la educación de calidad que brinda una institución de excelencia es, sobre todo, la que ayuda a los estudiantes: en el avance del espíritu: en su maduración integral, que provoca a seguir educándose, que afina la inteligencia y la voluntad, que orienta los afectos. Educación de calidad es también la que ayuda y obtiene logros de socialización y culturización. En fin, la que hace que los estudiantes "sepan y aprendan" muchas cosas y tengan capacidad y ánimo para seguir aprendiendo. Esto supone que debe haber, en una buena evaluación, pruebas o verificaciones de lo que se sabe y de los valores y actitudes que se tienen en diversos aspectos, cosas que no se suele considerar en las acreditaciones. Es decir, no se trata solamente de verificar sihay una buena gestión.

Los verificadores y la gestión preconizados nunca pueden llegar a todos los aspectos de la verdadera calidad educativa personal e institucional, en particular si no corresponden a un esquema integral, tal cual parece ser la matriz del IPEBA para la Educación Básica.

4. Lineamientos legales y normativos de la Evaluación, Acreditación y Calídad Educativa. Comentarios u observaciones

A continuación una revisión somera de lo normativo y legal que guía la calidad educativa, los verificadores, el SINEACE y su órgano operador el IPEBA. Ello permite descubrir los linean1ientos a partir de citas textuales vigentes. Asin1ismo, algunos comentarios u observaciones cuando se estime pertinente. Va organizado en cinco temas sobre los que parece necesario llamar especialmente la atención (de la letra A a la letra E).

A) Garantía únicamente de factores y funciona miento del SINEACE y sus organismos

1. "Corresponde al Estado garantizar los factores de la calidad en las Instituciones Públicas. En las Instituciones Privadas los regula y supervisa (Ley General de Educación, art.13).

2. "El Estado garantiza el funcionan1iento de un Sistema Nacional de Evaluación Acreditación y Certificación de la calidad educativa (SINEACE) que abarca..." (Ley General de Educación, art .14).

Comentarios u observaciones a los números1y 2.

La Ley General de Educación solamente garantiza dos cosas:

a) Los factores de la calidad en las instituciones educativas públicas (Ley General de Educa ción,art.13).

b) El funcionamiento del SINEACE y sus organismos (Ley General de Educación, art.14).

Por tanto, no se está garantizando calidad 
educativa integral (es decir de aspectos cognitivos y no cognitivos; así como los de gestión). Esto es muy lógico ya que es imposible que se pretenda garantizar una calidad integral de la educación; se puede promover y ayudar, pero no garantizar.

B) Promoción y garantía de calidad por el SINEACE ysus organismos

3. Entre las varias funciones de los órganos del SINEACE está la de promover: "Promueven una cultura de calidad entre los docentes y las instituciones educativas" (Ley General de Educación, art.16, inciso a).

4. Los organismos del SINEACE están "destinados a definir y establecer los criterios, estándares y procesos de evaluación, acreditación y certificación a fin de asegurar los niveles básicos de calidad que deben brindar las instituciones..." (Ley del SINEACE, art. 2).

5. El artículo 5 dice que el SINEACE "tiene la finalidad de "garantizar a la sociedad que las instituciones públicas y privadas ofrezcan un servicio de calidad" (Ley de SINEACE, art.5).

6. Al definir a los órganos operadores del SINEACE, se dice: "Los órganos operadores son los encargados de garantizar la calidad educativa en elámbito..." (Ley del SINEACE, art.13).

Entre los objetivos de los órganos operadores está: "Garantizar la óptima calidad de las instituciones educativas... fomentando..." (Ley del SINEACE, art.17).

También, "Promover y contribuir a la continua elevación de la calidad..." (Ley del SINEACE, art.17).

Al definir la Ley del SINEACE lo que es el IPEBA, se dice: "El IPEBA es el órgano operador encargado de definir los estándares de medición internos e indicadores para garantizar en las instituciones... los niveles aceptables de calidad educativa..." (Ley del SINEACE, art. 21).

7. En el Reglamento del SINEACE, al señalar las funciones específicas del mismo (art. 4) y siguiendoloexpresado en el Art.1del mismo reglamento, se dice: "Aprobar los lineamientos de política para el funcionamiento delSINEACEylosórganos operadores, a fin de garantizar la calidad del sistema educativo peruano en su conjunto" (ReglamentodelSINEACE, art.4,incisob).

Comentario u observaciones a los números 3a 7:

En momentos, el Estado parece colocarse más bien en la línea lógica de "promover" la calidad educativa (Ley General de Educación) . En otros, más bien en la línea más discutible de garantizar (SINEACE e IPEBA). Unas veces se refiere a las instituciones educativas; otras, a todo el sistema educativo; en casos aparenta abarcar la calidad integral (lo cognitivo, no cognitivo y de gestión); en otros momentos solamente aspectos de servicio y gestión. En el fondo, se estima que los organismos del SINEACE van a ser los hacedores de la calidad educativa, cosa imposible. Así, entre otros:

La Ley del SINEACE va más allá de la Ley General de Educación y del sentido común al manifestar que sus organismos están destinados a "asegurar los niveles básicos de calidad" (Ley del SINEACE, art . 2). Como quiera que esta ley sea posterior a la Ley General de Educación, prevalece. En consecuencia, se pasa de garantizar factores y elfuncionamientodel SINEACE (art.13 y14 de la Ley General de Educación) a "asegurar los niveles básicos de calidad" por parte de los órganos del SINEACE, lo cual constituye un paso muy largo e ilógico que parece imposible cumplir.

Además, y sobre todo, supone un esquema de calidad educativa integral, como debe ser, cosa en la que no coincide la matriz del IPEBA que se reduce a la gestión de las instituciones educativas.

Sin embargo, la misma ley delSINEACE es un poco más modesta en el art.5, en donde lo que se garantiza es solamente un servicio de calidad, y no toda la calidad educativa.

En las citas de la Ley del SINEACE se hace referencia a que los órganos operadores están para garantizar la calidad educativa en las institucione" educativas. También, está la idea de promover la calidad y la definición de 
estándares e indicadores.

Es visible que aquí se da un paso claro más hacia la idea de que los órganos están destinados a garantizar la calidad no solamente de la gestión (tal como aparece en la matriz dentro de un recorte no correcto), sino la calidad integral que va más allá de la gestión (logros de todo tipo de aprendizaje: cognitivos y no cognitivos).

EN SÍNTESIS: Hay claras contradicciones legales y normativas (ley General, ley del SINEACE, Reglamento y Matriz) en lo fundamental y una gran utopía en varios casos.

Por otra parte, no se ve claro cuál debe ser el contenido de la evaluación y acreditación: ¿abarca toda la calidad educativa? Y si abarca solamente una parte (la gestión), ¿vale la pena tanto esfuerzo? En verdad debiera abarcar la calidad integral, pero sin garantizar en forma absoluta nada, dejando libertad siempre para la Acreditación tal como se está planteando.

\section{C) Objetivo y foco dela Evaluación y Acreditación}

8. la ley General de Educación en relación con las funciones del SINEACE y sus organismos, dice: "Evalúan, en los ámbitos regional y nacional, la calidad de los aprendizajes y delos procesos pedagógicos y de gestión" (ley General de Educación, Art . 16, inciso e). Es la misma línea de la ley del SINEACE cuando en su Art. 2 habla de que los organismos (del SINEACE) "establecen criterios... a fin de asegurar los niveles básicos de calidad que deben brindar las instituciones..."

9. Sobre los procesos de evaluación para el mejoramiento de la calidad educativa (ley del SINEACE, capítulo lll), se dice: "Autoevaluación de la gestión pedagógica, institucional y administrativa, que está a cargo de los propios actores de la institución educativa" (Ley delSINEACE, art.ll).

10. la matriz del IPEBA ("Matriz de evaluación para la acreditación de la calidad de la gestión educativa de instituciones de Educación Básica Regular", 201l), dice en la introducción: "En la segunda parte se hacen explícitos los propósitos de la acreditación, se describe brevemente cada etapa del proceso y se delimita el foco de evaluación: la gestión educativa. Es decir, la capacidad que tiene la institución para dirigir los procesos, recursos y toma de decisiones en función de la mejora permanente..." (Introducción MatrizdelIPEBA, 2011).

Comentarios u observaciones a los números 8 a10:

Se observa que el citado $\mathrm{n}^{\circ} 8$ comprende toda (no solamente la gestión). Así, incluye en primer lugar, la calidad de los aprendizajes (se entiende los conocimientos, valores, desarrollo de capacidades, competencias, en el fondo lo cognitivo y lo no cognitivo). También, la idea de asegurar los niveles básicos de calidad. Se trata de un concepto amplio, no reductivo, no limitado a la gestión.

Queda claro que, en la 2da. y 3ra. citas ( $n^{\circ} 9$ y 10), que lo que se solicita es solo la gestión, lo que lo hace en verdad un esquema reductivo, al no estar contempladas evaluaciones sobre avances en conocimientos, capacidades, competencias y, aunque se diga que en estas últimas está toda la riqueza educativa, es visible que ética, valores, religiosidad y gran parte delo que se pudiera llamar espíritu dela persona, falta. Es decir, se evalúa solamente la gestión. Si sólo se evalúa la gestión, los factores, estándares e indicadores, lógicamente, irán únicamente a ella. Es decir que, las dos últimas citas muestran con claridad que el objetivo y foco de la matriz es lagestiónynotambiénloslogros cognitivosy no cognitivos necesarios para una calidad integral.

D) libertad deAcreditación

11. El SINEACE y sus organismos (ejemplo, el IPEBA:), "Acreditan periódicamente la calidad de sus instituciones públicas y privadas" (Ley General de Educación, art . 16, inciso c).

El art. 12 de la ley del SINEACE manifiesta, textualmente, el carácter voluntario de la Acreditación: "la evaluación con fines de Acreditación tiene carácter voluntario . El reglamento de la presente ley regula el proceso de evaluación externa, así como la vigencia de la acreditación y los casos en que éstas son obligatorias" (Ley delSINEACE, art.12). 
Comentarios u observaciones al número11:

El sentido de la Ley General de Educación en su Art. 16, inciso c, es que la Acreditación es obligatoria para las instituciones educativas de todos los niveles (Ley General de Educación, Art. 16, letra c). No obstante, la Ley del SINEACE dice que es voluntaria (salvo en algunos casos del nivel superior). Como es una ley posterior al General de Educación, se entiende que prevalece. Además, la Acreditación suele ser siempre un proceso voluntario y no obligatorio. Es evidente que la Ley de Educación debiera modificarse en su Art. 16, inciso c. O de lo contrarío, que se aclare, ya que parece haber una flagrante contradicción.

\section{E) Libertad de Enseñanza y Acreditación}

12. Varias citas pueden ayudar a reflexionar sobre este tema. Así, en la Constitución (1993) se dice:

"El Estado reconoce y garantiza la libertad de enseñanza" (Constitución,art.13).

"La enseñanza se imparte, en todos sus niveles, con sujeción a los principios constitucionales y a los fines de la propia institución educativa" (Constitución, art.14).

"El Estado coordina la política educativa. Formula los lineamientos generales de los planes de estudio, así como los requisitos mínimos de la organización de los centros educativos" (Constitución, art.16).

"El Estado garantiza la libertad de cátedra y rechaza la intolerancia" (Constitución, art. 16).

"Cada universidad es autónoma en su régimen normativo, de gobierno, administrativo y económico (Constitución, art.16).

13. La Ley de centros educativos privados, en sus artículos 3 y 5 , señala el control y guía general de los promotores en sus centros educativos, incluyendo lo pedagógico y académico (Ley 26549, 1995).

14. El Decreto Legislativo NQ 882 (1996) en el art. 5, señala también los derechos de los promotores de las instituciones educativas privadas sobre los más variados aspectos de su institución, en cualquier nivel educativo al que corresponda.

15. El sistema de Acreditación establecido señala, en la línea que podríamos llamar de riesgo para la libertad de enseñanza, lo siguiente:

"El órgano operador del SINEACE (IPEBA u otro) establece, publica y pone a disposición de las instituciones y programas educativos los estándares, criterios, indicadores y procedimientos de evaluación, así como los requerimientos de evidencia documentaría que deben servir de fuente de verificación del contenido del informe de autoevaluación" (Reglamento de la Ley del SINEACE, art. 15.1).

"Cuando la autoevaluación se realiza con fines de acreditación, la institución o programa utilizará los estándares, criterios y procedimientos aprobados por el órgano operador correspondiente" (Reglamento de la Ley del SINEACE, art.12.3).

Comentarios u observaciones a los números 12 a 16:

En las primeras citas transcritas se percibe que la libertad de enseñanza es muy clara en el Perú y más aún para las instituciones privadas. La impresión que se tiene es que el sistema de Acreditación (ver referencias del nQ 15), recién citado, contradice de manera importante lamencionada libertad.

Si para acreditarse hay que utilizar obligatoriamente solamente lo que señala la normatividad analizada: factores, estándares, etc.; es decir, lo que establece el órgano correspondiente del SINEACE (IPEBA), se le ha quitado a la institución educativa la posibilidad de acreditarse según su propia cosmovisión pedagógica, ética y valórica, etc. Es obvio que la libertad de enseñanza constitucional no está funcionando.

Por todo lo anterior, las instituciones educativas deben tener derecho a escoger algunos criterios o factores propios, así como estándares e indicadores peculiares de su misión, objtcivos, y otros, aparte de los señalados por el IPEBA. Asimismo, poder 
incluir aspectos cognitivos y no cognitivos varios y no tener que quedar reducidas a una evaluación de solamente la gestión.

CONCLUSIONES sobre aspectos legales y normativos del número 4 :

1.- El objetivo de la evaluación propuesto por el IPEBA no es la calidad integral de la educación, sino la gestión de lo pedagógico, administrativo e institucional, y sí bien ello es importantísimo, y parece muy bien elaborado lo realizado al respecto por el IPEBA, la evaluación y acreditación debe ir a la integralidad de la calidad, por tanto también a los aspectos cognitivos y no cognitivos.

2.- No se puede nunca garantizar la calidad de la educación. Mucho menos aún si la evaluación propuesta no es integral y es más bien reductíva como en este caso, al dejar ele lado aspectos de suma importancia que tiene que ver con logros reales en lo cognitivo y no cognitivo.

3.- Es adecuado que las instituciones y programas sean libres de acreditarse o no, más cuanto son tantas (unas 90,000) que va a ser prácticamente imposible acreditarlas (a menos se haga superficialmente). Además, el costo y recursos humanos que requiere lo harán imposible para no pocas instituciones educativas. Habrá que ver si el Estado evalúa y acredita a los suyos o queda como una disposición para los demás.

4.- Parece claro que la imposición ele parámetros gubernamentales va a generar, o quiere generar, un MODELO ÚNICO de institución educativa en todos los niveles, lo cual, aparte de estar reñido con el principio de libertad de enseñanza, va a recortar la creatividad, la innovación, y otros, vistos ya cuáles son los factores, estándares e indicadores quese vanproponiendo, omejor digamos imponiendo, para que una institución pueda ser acreditada. Alhacerlo, se quiere aprovechar el deseo de acreditarse de las instituciones educativas, seguramente por deseo de marketing en no pocos casos, paraimponerlesun esquema que deja delado los aspectos cognitivos y no cognitivos y se hipoteca solamente a los de gestión.
5.- El establecer mediante el SINEACE y sus órganos operativos: factores, estándares, indicadores de evaluación, y otros, para la certificación y acreditación ¿̇asegurará o garantizará la calidad? Parece cuestionable. Aparte de que las leyes y normas no generan calidad (lo hacen el trabajo abnegado, técnico y afectuoso de padres y maestros y la respuesta generosa y esforzada de los alumnos), un examen, incluso superficial, muestra que los instrumentos, por sofisticados que sean, nunca pueden valorar ni medianamente a una persona o a una institución, menos aún predecir su actuar futuro.

6.- El planteamiento del propósito del sistema, que el SINEACE garantiza la calidad, debió ser más modesto (no puede "asegurar" la calidad, sino ser un tesonero y valioso colaborador). Además, y sobre todo, debió hacer visible y pedir la colaboración de otros agentes clave, particularmentela familia y el propio estudiante (al docente sí se le menciona adecuadamente al hablar de los factores). Parecería que el Sistema garantiza por sí mismo e independientemente de los agentes fundamentales, lo cual no es ni cierto ni posible.

7.- Asimismo, se debe tener en cuenta que, al suponer que los órganos y verificadores son los que conseguirán las calidad, aparte de no ser cierto, les otorga fácilmente una fuerza y autoridad que nos les corresponde y que se derivará en directivas y planteamientos obligatorios y exigentes que, fácilmente, atentarán contra la libertad de enseñanza y correcta autonomía de las instituciones educativas.

\section{IPEBA: MATRIZ DE EVALUACIÓN PARA LA ACREDITACIÓN}

\section{Análisis y observaciones}

En mayo de 2011 se ha publicado por el IPEBA (Instituto Peruano de Evaluación, Acreditación de la Calidad de la Educación Básica), un documento sustantivo de su trabajo: "Matriz de Evaluación para la Acreditación de la Calidad de la Gestión Educativa de las Instituciones de Educación Básica Regular".

Dicho documento, juntamente con otros que se 
han publicado y publicarán, tiene como objeto: orientar la "implementación del proceso de acr edit aci ón ... que guiará el proceso de autoevaluación y la elaboración e implementación deplanes demejora..."

En la primera parte se presenta el concepto de calidad de la educación que sustenta la matriz. En la segunda parte se explicitan los propósitos de la acreditación y se delimita el foco de evaluación: la gestión educativa. En la tercera parte se describen los cinco factores clave a evaluar. En la cuarta y última se detalla la matriz deevaluación.

\section{Análisis}

Sin desmerecer el esfuerzo y los méritos (que los hay y muy significativos), la matriz de evaluación permite observar una prioridad notable a los aspectos de gestión, tal cual corresponde a su título. Pero si consideramos que la Calidad Educativa va más allá de los aspectos de gestión, encontramos ausencias o vacíos que se van a tratar de señalar a continuación.

a) Sepuedemuybientenerlaimpresiónquelos estándares establecidos van básicamente en la línea cognitiva, tanto en lo que atañe a la enseñanza-aprendizaje como en las prácticas educativas, pero no es así: están orientados centralmente a la gestión. Por otra parte, lo no cognitivo está ausente, aunque se pueda decir que en las competencias estátodo.

b) Así como con toda razón se establecen, y en casos con insistencia, palabras significativas como: equidad, inclusión, diversidad, conocimientos, habilidades, participación, y otras, en cambio no se menciona ni enfatiza términos venerables como: ética, moral ciudadanía, espiritualidad, religiosidad. La misma palabra valores solamente se menciona una sola vez en lo que se refiere a estándares e indicadores. Esto quiere decir que a estos aspectos no se les da importancia, siendo así que constituyen un elemento clave en una educación integral y por tanto en estándares eindicadores escolares.

c) La matriz que se presenta es una "matriz de evaluación de la gestión de la calidad de la educación", no de logros en conocimientos y aspectos no cognitivos, como si evaluando y acreditando solamente la gestión fuera suficiente para saber sí hay calidad en una institución educativa y en los estudiantes. Es decir, permitiría pensar que si la gestión es buena o mala, la calidad de los aprendizajes y la enseñanza será de uno u otro tipo. A fin de cuentas, siendo la gestión importantísima y clave, es solamente una parte de la institución educativa y del logro deseado.

d) Lo importante para saber si un centro tiene realmente calidad educativa es verificar, dentro de lo posible y difícil que es esto, si hay logros reales en los aprendizajes (desde luego tanto cognitivos como no cognitivos). En la matriz, ni los unos ni los otros son objeto de evaluación ni significan algo en las evaluaciones de las acreditaciones, pese a que ellos mismos constituyen el centro de las preocupaciones educativas. Lo más que se menciona son las informaciones genéricas que presentan el Ministerio de Educación, las pruebas PISA, y otras. Puesto de diferente forma, el saber que hay una buena o mala gestión no garantiza malos o buenos resultados en losaprendizajes .

\section{Comentarios u observaciones a la $\mathrm{N}^{0} 5$ Matriz de} Evaluación

l. El Estado no puede garantizar la calidad educativa mediante el SINEACE o el IPEBA; puede ayudar y promoverla. No hay que ofrecer lo que no se puede dar. Se corre el riesgo de colaborar a una frustración.

2. La matriz comentada del IPEBA es insuficiente al prescindir de aspectos importantes de los logros cognitivos y lo espiritual en el amplio sentido de la palabra; al evaluar solamente la gestión, y dar únicamente indicativos genéricos en cuanto a los conocimientos. (La publicación de posterior documentación del IPEBA debiera mejorar esta observación, aunque no es muy seguro ya que el Art. 11 de la Ley del SINEACE y la introducción de la matriz del IPEBA comentada establecen como foco la evaluación dela gestión y no los logros).

3. La matriz del IPEBA da la clara impresión de que está colisionando con el principio de la libertad de ...:nseñanza y autonomía de los centros, ya que establece un modelo rígido de 
factores y estándares que, con las falencias indicadas, no sería adecuado para muchas instituciones educativas debido a su línea axiológica, pedagógica, o de fuerte raigambre espiritual.

4. Cada institución educativa debiera tener derecho a evaluarse y acreditarse según factores y estándares que van de acuerdo a su Proyecto Educativo Institucional, sin negar otros señalados por elIPEBA.

S. En el desarrollo de la matriz hay aspectos muy valiosos en el área de gestión que es preciso resaltar y felicitar, pero que no son objeto de este trabajo más bien destinado a observar las falencias o problemas.

\section{CONCLUSIONES GENERALES}

La Evaluación, Acreditación y Certificación de la Calidad Educativa, en proceso de implementación por el Estado mediante los organismos correspondientes del SINEACE, constituye un aporte valioso y va a colaborar a la mejora de la calidad en el Perú.

No obstante:

a) Hay que evitar verla o presentarla como algo que de por sí, y de manera casi mágica, va a resolver el problema de la calidad educativa en los distintos niveles del sistema educativo peruano. Esto constituiría una utopía peligrosa que podría terminar en frustración nacional más.

b) Mediante el SINEACE y la implementación de sus organismos no es posible garantizar la Calidad Educativa. Se puede sí colaborar y promover a su obtención.

c) La evaluación y Acreditación de la Calidad Educativa en las instituciones educativas, en este caso las que tienen relación con el IPEBA, para ser integral como se estípula, debe comprender los más variados aspectos: cognitivos, no cognitivos y de gestión. Ello supone: evaluación de logros en conocimientos, valores, actitudes, sentido ético, religioso, y otros; así como constancia de desarrollo de capacidades, competencias; igualmente una gestión satisfactoria.

d) La matriz presentada por el IPEBA se centra en aspectos de la gestión, por lo que se le observa reductiva $\mathrm{y}$, por tanto, insuficiente para verificar la calidad de una institución educativa.

e) La libertad de enseñanza queda seriamente cuestionada ante una Acreditación, se establece solamente en base a estándares, y otros, señalados por el IPEBA.

\section{RECOMENDACIONES}

Que el SINEACE y sus organismos presenten un esquema de Calidad Integral y que, por tanto, la matriz del IPEBA no enfatice tanto la gestión descuidando los otros aspectos de logros y avances cognitivos yno cognitivos.

Que la matriz del IPEBA permita y promueva que las instituciones educativas coloquen estándares, factores e indicadores que tienen relación con su peculiaridad educativa. Esto parece muy lógico y resguarda el principio constitucional de libertad de enseñanza.

Que no se insista en que los diversos procesos constituyen una garantía de calidad educativa, evitando así las utopías educativas que son muy peligrosas y frustran a las personas y al país.

Que en la Evaluación y Acreditación se incluyan pruebas a los estudiantes que permitan detectar el nivel de logros de aprendizajes en aspectos cognitivos y no cognitivos.

Que se hagan las modificaciones legales necesarias para que no haya contradicciones, espacialmente entre la Ley General de Educación y la del SINEACE.

\section{NOTAS}

(1) Este articulo está referido a la etapa de Educación Básica.

(2) Resultados exitosos tiene que ver con avances no sólo en los conocimientos, habilidades y otros, sino también en todas las otras

\section{REFERENCIAS}

Constitución Política del Perú (1993).

DecretoSupremo Nº18-2007-ED. Reglamento dela Ley del SINEACE. IPEBA (2011). Matriz de Evaluación para la Acreditación de la calidad de lagestión educativa deinstituciones de Educación Básica Regular.

facetas personales y sociales del estudiante.

(3) La letra negrita, en todo el texto, siempre es del articulista.

Coordinador de la Maestría de Educación de UNIFÉ (2006 al 2011). Doctor en Educación por la Pontificia Universidad Católica del Perú. Exprofesor del Posgrado enuniversidades yexdirector decolegios. Expresidente delConsorcio de "Centros Educativos Católicos. última publicación: "Política Educativa Escolaren el Perú" (2006), Lima.

Ley General de Educación №18044 (2003).

Ley del Proyecto Educativo Nacional (2007) LeydelSINEACE N²8740 (2006). 